\title{
Effects of varying Notch1 signal strength on embryogenesis and vasculogenesis in compound mutant heterozygotes
}

\author{
Changhui $\mathrm{Ge}^{1,2}$ and Pamela Stanley*1
}

\begin{abstract}
Background: Identifying developmental processes regulated by Notch1 can be addressed in part by characterizing mice with graded levels of Notch1 signaling strength. Here we examine development in embryos expressing various combinations of Notch 1 mutant alleles. Mice homozygous for the hypomorphic Notch $1{ }^{12 f}$ allele, which removes the single O-fucose glycan in epidermal growth factor-like repeat 12 (EGF12) of the Notch1 ligand binding domain (Ibd), exhibit reduced growth after weaning and defective T cell development. Mice homozygous for the inactive Notch $7^{1 b d}$ allele express Notch1 missing an 20 kDa internal segment including the canonical Notch1 ligand binding domain, and die at embryonic day $\sim$ E9.5. The embryonic and vascular phenotypes of compound heterozygous Notch112f/lbd embryos were compared with Notch 1+/12f, Notch 112f/12f, and Notch 1lbd/lbd embryos. Embryonic stem (ES) cells derived from these embryos were also examined in Notch signaling assays. While Notch1 signaling was stronger in Notch 1 12f/lbd compound heterozygotes compared to Notch 1 lbd/lbd embryos and ES cells, Notch1 signaling was even stronger in embryos carrying Notch112f and a null Notch1 allele.
\end{abstract}

Results: Mouse embryos expressing the hypomorphic Notch $12{ }^{12 f}$ allele, in combination with the inactive Notch $1^{1 b d}$ allele which lacks the Notch1 ligand binding domain, died at E11.5-12.5. Notch112f/lbd ES cells signaled less well than Notch 112f/12fES cells but more strongly than Notch 1/bd/lbd ES cells. However, vascular defects in Notch 112f/lbd yolk sac were severe and similar to Notch 1/bd/lbd yolk sac. By contrast, vascular disorganization was milder in Notch 112f/lbd compared to Notch 1/bd/lbd embryos. The expression of Notch1 target genes was low in Notch112f/lbd yolk sac and embryo head, whereas Vegf and Vegfr2 transcripts were increased. The severity of the compound heterozygous Notch 112f/lbd yolk sac phenotype suggested that the allelic products may functionally interact. By contrast, compound heterozygotes with

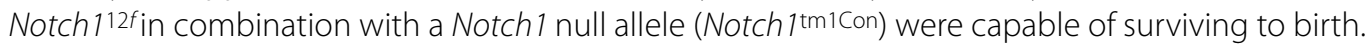

Conclusions: Notch 1 signaling in Notch $12 f / 16 d$ compound heterozygous embryos is more defective than in compound heterozygotes expressing a hypomorphic Notch $112 f$ allele and a Notch 1 null allele. The data suggest that the gene products Notch $1^{\text {bd }}$ and Notch $1{ }^{12 f}$ interact to reduce the activity of Notch $1{ }^{12 f}$.

\section{Background}

Notch transmembrane receptors are important regulators of cell fate determination in numerous cell types [13]. Notch receptors in Drosophila and mammals are covalently modified with $O$-fucose on many epidermal growth factor-like (EGF) repeats of the extracellular domain [4]. An important $O$-fucose site resides in epider-

* Correspondence: pamela.stanley@einstein.yu.edu

1 Department of Cell Biology, Albert Einstein College of Medicine, New York, NY-10461, USA

Full list of author information is available at the end of the article mal growth factor-like repeat 12 (EGF12) which, together with EGF11, is required for canonical Notch ligand binding to Drosophila Notch [5-7] and to mammalian Notch1 [8]. A point mutation that precludes the addition of fucose to EGF12 in Drosophila Notch results in enhanced binding of both Delta and Serrate Notch ligands, and a hyperactive Notch that is refractory to Fringe [9]. However, the same mutation (Notch112f) in cultured mammalian cells gives markedly reduced signaling in a Notch reporter signaling assay $[10,11]$, predicting a Notch1 null phenotype in vivo. Surprisingly however, homozygous 
Notch $122 f 12 f$ mice are viable and fertile, but exhibit retarded growth and mild defects in $\mathrm{T}$ cell development in the thymus [12], consistent with weak Notch1 signaling. Notch $1^{+/ 12 f}$ heterozygotes are indistinguishable from wild type in terms of growth and $\mathrm{T}$ cell development. However, compound heterozygotes carrying Notch $1^{12 f}$ and the inactive Notch $1^{l b d}$ allele, which lacks the ligand binding domain and generates an inactive $\sim 280 \mathrm{kDa}$ Notch1 receptor at the cell surface, are not born [12]. Therefore Notch $12 f$ is a hypomorphic allele in mammals and the $O$-fucose glycan in the ligand binding domain is required for optimal Notch1 signaling. Homozygous Notch $1^{\text {lbdllbd }}$ embryos die at $\sim$ E9.5 $[8,12]$ with an indistinguishable phenotype compared to Notch1 null embryos

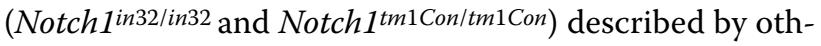
ers $[13,14]$. Heterozygous Notch $1^{+/ l b d}$ and Notch1+/tm1Con mice are viable and fertile whereas Notch 1 12fllbd compound heterozygotes die between E11.5 and E12.5, significantly later than either Notch1lbdllbd [12] or Notch1 null embryos [13,14] that do not express Notch1 [15-17].

The availability of these Notch1 mutant alleles suggested a genetic approach to determining effects of varying Notch1 signaling strength. The Notch $1^{\text {lbd }}$ mutation generates a non-functional but cell surface-expressed Notch1 that cannot signal $[8,12]$. Notch $1^{\text {tm } 1 \text { Con }}$ lacks Notch 1 on the cell surface due to the absence of its transmembrane domain [14]. Notch $1^{\text {in } 32}$ homozygous embryos have no Notch1 transcripts [13] and an indistinguishable phenotype from Notch1 $1^{\text {tm } 1 \text { Con }}$ homozygotes which lack Notch1 based on western analyses [15,18]. Notch1+/heterozygotes carrying either of the Notch1 null alleles exhibit Notch1 signaling defects in certain cell types, an effect attributed to Notch1 haploinsufficiency rather than to a dominant negative effect in Notch1 ${ }^{\text {tm } 1 \text { Con }}$ [18-21]. In this paper we compare embryogenesis and vasculogenesis in compound heterozygotes expressing the hypomorphic Notch $1^{12 f}$ allele with either the inactive Notch $1^{\text {lbd }}$ allele $[8,12]$ or the Notch $1^{\text {tm1 Con }}$ null allele [14].

\section{Results}

\section{Notch signaling in Notch 1 12f/lbd compound heterozygous} ES cells

The Notch $1^{12 f}$ and Notch ${ }^{l b d}$ alleles investigated here are diagrammed in Fig. $1 \mathrm{~A}$ and $1 \mathrm{~B}$ and their identification by PCR genotyping is shown in Fig. 1C. Previous studies showed that Notch $1{ }^{12 f l b d}$ compound heterozygotes die by $\sim$ E12.5 [12]. To examine Notch ligand binding and the strength of Notch signaling in more detail, ES cells were derived from Notch $1^{12 f l b d}$ compound heterozygous blastocysts and compared to ES cells derived from Notch112f/ $12 f$ and Notch $1^{\text {lbdlllbd }}$ homozygous blastocysts and wild type ES cells (Fig. 2). All cell lines bound the anti-Notch1

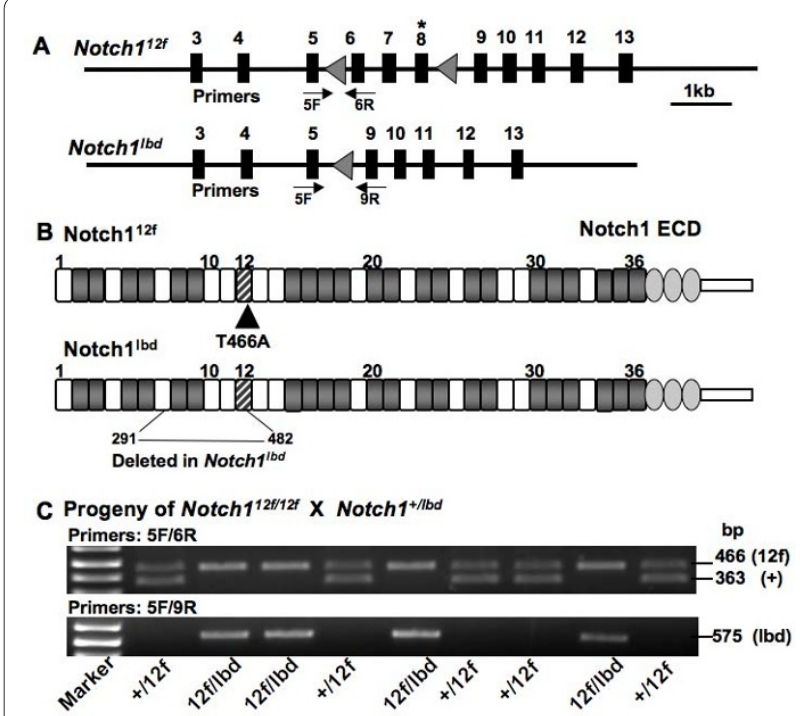

Figure 1 Generation of Notch $1^{12 f / l b d}$ embryos. (A) Diagram of the Notch $112 f$ and Notch 1 lbd alleles. (B) Diagram of mouse Notch1 EGF repeats in Notch $112 f$ and Notch 1 lbd extracellular domains. The EGF repeats with putative $\mathrm{O}$-fucosylation sites are shaded in gray and the mutation in EGF12 is shown. (C) Genotyping by PCR from E9.5 yolk sac DNA of a litter from a Notch $12 f / 12 f \times$ Notch $1+/ / b d$ cross. Primers $5 F$ and $6 R$ detect the Notch $12 f$ and Notch $1+$ alleles, primers $5 \mathrm{~F}$ and $9 \mathrm{R}$ detect the Notch $1^{\text {lod }}$ allele.

extracellular domain mAb 8G10 equivalently, and therefore expressed the various Notch 1 molecules similarly at the cell surface (Fig. 2A). Each mutant line exhibited a decrease in the low level of soluble Delta1 binding observed with wild type ES cells (Fig. 2B). Binding of Delta1 is not reduced to zero even in Notch1 null ES cells because of the presence of Notch2, Notch3 and Notch4 [17]. Notch signaling was analysed in co-culture assays with L cells or L cells expressing full length Delta1 or Jagged1 ligand. This reporter assay revealed a graded reduction in Notch signaling with Notch112f/12f $>$ Notch112fllbd $>$ Notch1 $1^{l b d l l b d}$ ES cells (Fig. 2C-D). This graded response was also observed by western analysis using Notch1 antibody Val1744 [15] which detects the $\sim 110 \mathrm{kDa}$ Notch1 fragment generated by $\gamma$-secretase complex cleavage of Notch1. The level of activated Notch1 in Notch 112fllbd ES cells was less than in Notch112fl ${ }_{12 f}$ ES cells, which was lower than in control ES cells, while Notch1 ${ }^{\text {lbdlllbd }}$ ES cells had undetectable levels of activated Notch1 (Fig. 2E). Nevertheless, all ES cell populations, including Notch $1^{l b d / l b d}$ ES cells, expressed equivalent levels of full-length Notch1 (Fig. 2E). Taken together, these data indicate that Notch $1{ }^{12 f}$ and Notch $1^{\text {lbd }}$ expression and transit to the cell surface were similar to wild type Notch1, but Notch1 signaling was reduced in mutant cells: Notch $12 \mathrm{ff}$ signaling was sightly less than wild type; 


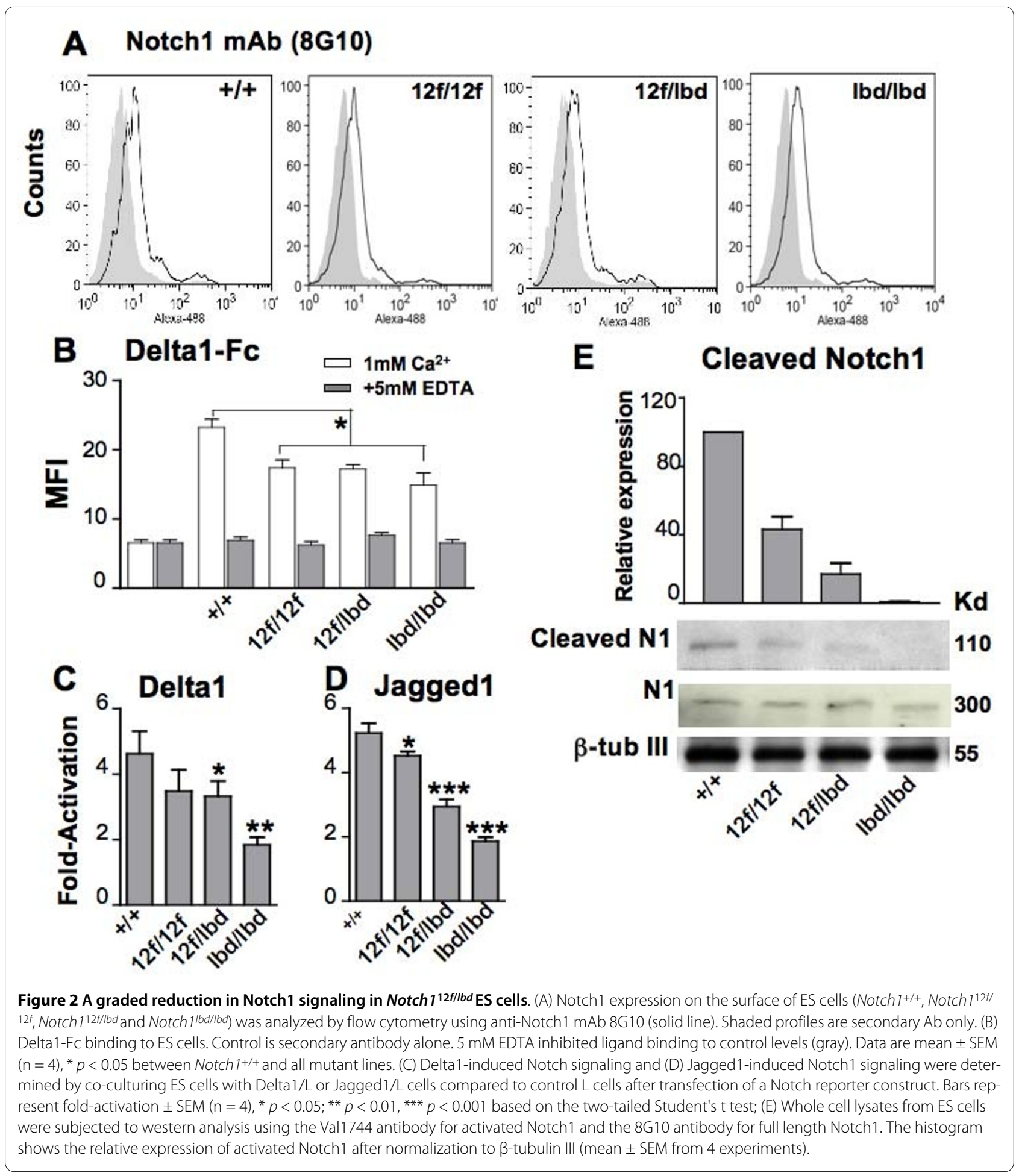

signaling from the combination of Notch $1^{12 f}$ and Notch $1^{\text {lbd }}$ was further reduced, and signaling by Notch $1^{\text {lbd }}$ alone was essentially absent. Previous experiments have shown that Notch $1^{l b d / l b d}$ and Notch $1^{\text {in } 32 / i n 32}$ ES cells which lack Notch1 $[13,15,16]$, are equally deficient in Delta1-Fc binding and Notch1 signaling [12].
Embryogenesis in Notch $1^{12 f / / b d}$ compound heterozygous embryos

Embryonic development was compared between Notch $1^{12 f / 12 f}$, Notch $12 f / l b d$ and Notch $1^{l b d / l b d}$ embryos. At E9.5 Notch112fllbd embryos formed 17-21 somites compared to 23-26 somites in Notch $1^{12 f / 12 f}$ embryos, the same 
as Notch1+/+ embryos, and 13-17 somites in Notch1 1 lbd/lbd embryos [8], the same as Notch1tm1Con null embryos [14] (Table 1). Compared to Notch $1^{12 f / 12 f}$ and Notch $1^{+/ 12 f}$ embryos, Notch112fllbd embryos also showed severely defective vasculogenesis in yolk sac at E9.5, similar to Notch $1^{\text {lbd/lbd }}$ yolk sac. By contrast, Notch112fllbd embryos at E9.5 and E10.5 exhibited milder defects in development than Notch1lbd/lbd embryos [12] (Fig. 3), although the ballooning of the pericardial sac and defective heart development were severe, and similar to mutants globally defective in Notch signaling such as mutants lacking Pofut1 [22], RBPJk [23] or presenilins 1 and 2 [24]. Taken together, these data indicate that two copies of Notch $12 f$ do not noticeably affect mouse embryogenesis at a gross level, whereas a single copy of Notch $1^{12 f}$ with Notch $1^{\text {lbd }}$ support embryonic development $\sim 2.0-2.5$ days longer than embryos with two copies of Notch $1^{l b d}$.

\section{Vasculogenesis in yolk sac appears to require stronger Notch 1 signaling than in the embryo}

Notch1 signaling is critical for vasculogenesis during mouse embryogenesis [25]. Loss of Notch1 in embryos [26] or in endothelial cells [27] causes embryonic lethality with severe vascularization defects in yolk sac, placenta and embryo. Blood that had leaked from the heart and blood vessels was apparent in Notch1 12 fllbd embryos (Fig. 3I-K; arrows). Vascular organization in embryos was examined by staining with anti-Pecam1 (endothelial marker platelet/endothelial cell adhesion molecule-1). Notch 12f/12f embryos (Fig. 4B, F, J, N) did not exhibit any apparent defects in brain, heart or intersomitic vascularization compared to Notch $1^{+/ 12 f}$ embryos. Notch 1 12f/lbd embryos exhibited somewhat disorganized vascularization in embryos, especially in the main trunk of the anterior cardinal vein, the vascular network of the head and heart, and in intersomitic vessels (Fig. 4C, G, K, O). Notch $1^{\text {lbd } / l b d}$ embryos exhibited severe defects in vascularization (Fig. 4D, H, L, P). Therefore, the extensive vascularization in E9.5 and older Notch12fllbd embryos appears to be well supported by the level of Notch1 signaling provided by the Notch $1^{12 f}$ allele. Considering that the vascular defects in yolk sac of compound heterozygous Notch $1^{12 f l l b d}$ and homozygous Notch $1^{l b d / l b d}$ embryos were similarly severe, the comparatively milder defects in Notch112fllbd embryos indicated that Notch1 signaling from a single copy of Notch 1 12f, while not sufficient to support vascularization in yolk sac at E9.5, is able to support a high level of vascularization in E9.5 embryos. It seems that vascularization in yolk sac requires stronger Notch1 signaling than in the embryo.

\section{Notch 1 target gene expression in E9.5 yolk sac versus embryo}

Whereas vascularization was severly affected in both yolk sac and embryo of Notch $1^{\text {lbd } / l b d}$ embryos, only the yolk sac of Notch $1^{12 f l b d}$ compound heterozygous embryos exhibited extremely defective vascularization. To investigate further, the expression of vasculogensis-related and Notch1 target genes was examined by real-time PCR using total RNA isolated from E10.5 Notch112fllbd and Notch $1^{+/ 12 f}$ yolk sacs and embryo heads. The relative expression levels of Pecam1 and Vegf were increased in Notch1 $1^{12 f l l b d}$ yolk sacs and embryos, and Vegfr2 expression was increased in Notch112fllbd embryo heads (Fig. 5AC). Therefore loss of Notch1 signaling upregulated transcription of the Pecam1, Vegf and Vegfr2 genes. Interestingly, the increased expression of Vegf and Vegfr 2 was greater in Notch112fllbd embryos, consistent with the relative strength of Notch1 signaling being greater in yolk sac. Expression of the Notch1 target genes Hes5, Hey1

Table 1: Somite Numbers in Notch 1 Mutants

\begin{tabular}{|c|c|c|c|}
\hline Genotype & Stage & No. Embryos & No. Somites \\
\hline$+/ 12$ f or $+/+$ & E9.5 & 8 & $23,23,24,24,24,25,25,26$ \\
\hline $12 f / 12 f$ & E9.5 & 4 & $23,24,25,26$ \\
\hline $12 \mathrm{f} / \mathrm{lbd}$ & E9.5 & 6 & $17,17,18,18,19,21$ \\
\hline $12 \mathrm{f} / \mathrm{tm} 1 \mathrm{Con}$ & E9.5 & 7 & $18,19,21,21,23,24,26$ \\
\hline $\mathrm{lbd} / \mathrm{lbd}$ & E9.5 & 6 & $13,14,14,16,17,17$ \\
\hline tm1Con/tm1Con & E9.5 & * & $\leq 14$ \\
\hline$+/ 12$ f or $+/+$ & E10.5 & 3 & $33,34,35$ \\
\hline $12 f / 12 f$ & E10.5 & 3 & $32,32,34$ \\
\hline $12 \mathrm{f} / \mathrm{lbd}$ & E10.5 & 5 & $18,18,21,22,23$ \\
\hline
\end{tabular}

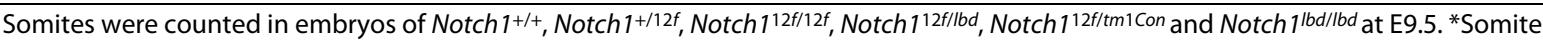
numbers in Notch $1 \mathrm{tm} 1 \mathrm{Con} / \mathrm{tm} 1$ Con embryos are from Conlon et al. [14]. 


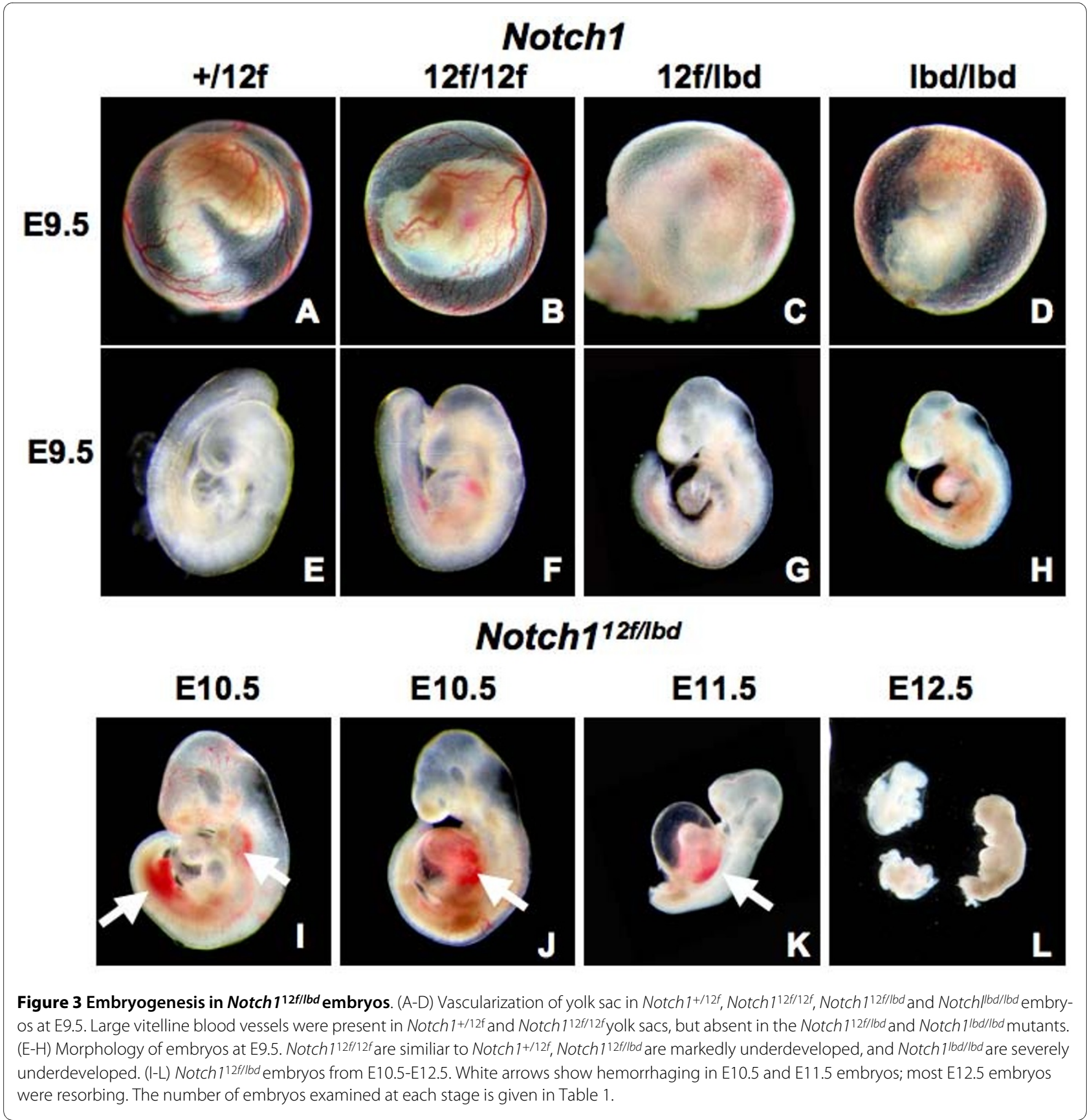

and Hey2 was reduced in Notch $1^{12 f l l b d}$ yolk sac (Fig. 5DF), but the level of Hes 1 transcripts was not changed (data not shown). In embryos, only the expression of Hes5 was significantly reduced compared to control. The expression of Ang1, Tie2 and Ephrin-B2 which are involved in angiogenesis, as well the expression of Notch1 itself, were not changed in Notch1 12fllbd yolk sac or embryos (data not shown). The fact that the increase in Vegf and Vegfr2 transcripts was more in embryo head than yolk sac $(418 \%$ vs $170 \%$ for Vegf; $227 \%$ vs. $148 \%$ for Vegfr2; Fig. 5B and $6 \mathrm{C})$, and the fact that the reduction in Notch target gene expression was greater in yolk sac than embryo head, correlated generally with Notch1 signal strength and the greater severity of vascularization defects in yolk sac versus embryo head.

Notch $1^{12 f}$ may function to birth in the absence of Notch $1^{\text {lbd }}$ The severity of the Notch112fllbd phenotype suggested an interaction between Notch $1^{12 \mathrm{f}}$ and Notch $1^{\text {lbd }}$ that interfered with signaling by Notch $1{ }^{12 f}$. In this case, compound heterozygous embryos expressing a Notch 122 allele and a Notch1 null allele might be expected to have a milder 


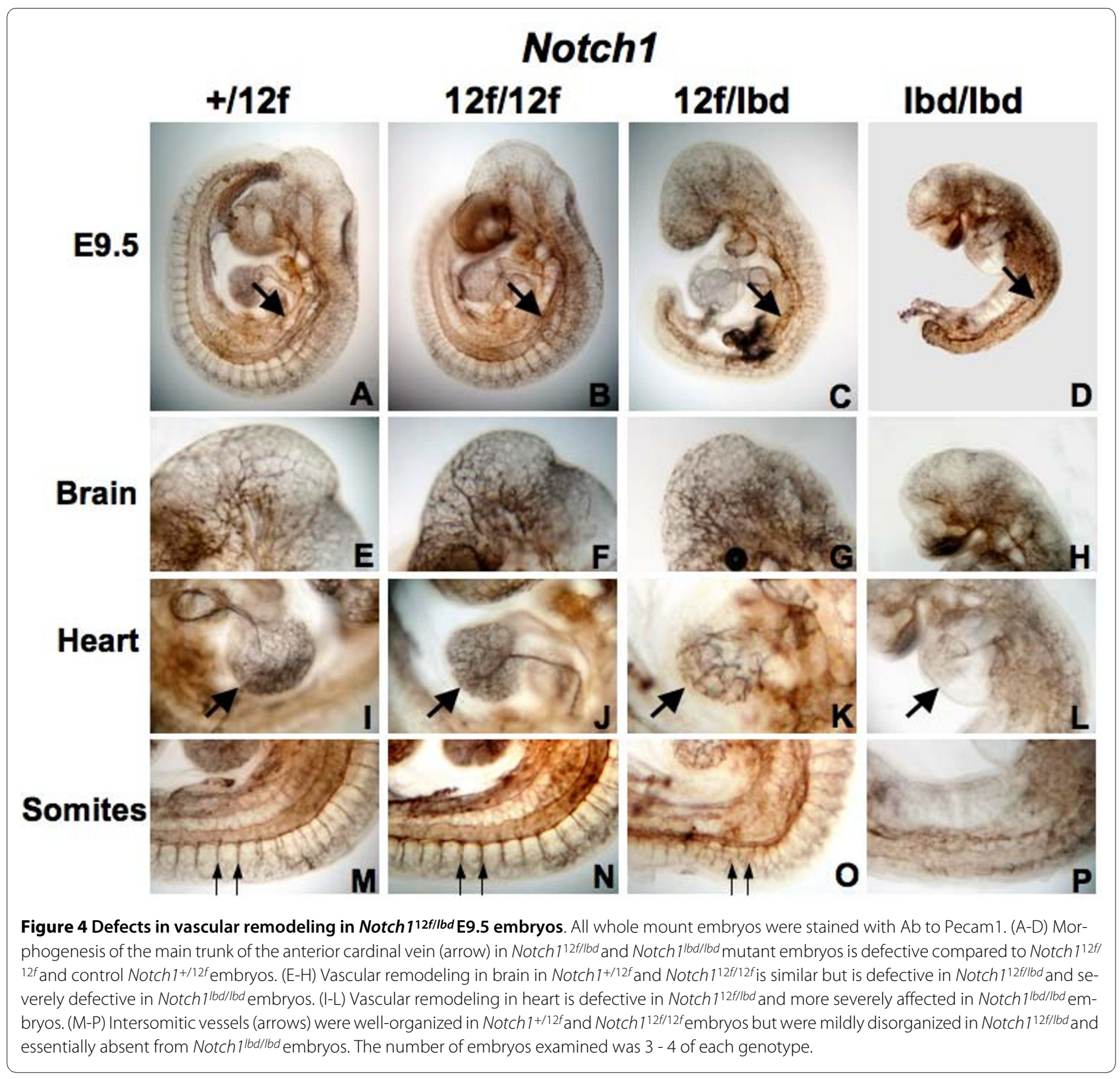

phenotype than Notch $1^{12 f l b d}$ embryos. Notch $1^{12 f / 12 f}$ mice were crossed with Notch1+/tm1Con heterozygotes and embryos were examined at E9.5 and later (Fig. 6, Table 2). Some Notch1 12fttm1 Con embryos died between E11.5 and E12.5 with similar defects to Notch112fllbd embryos. However, this is $\sim 1.5$ days later than observed with

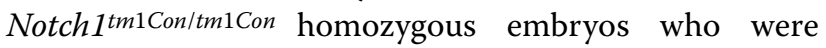
mostly dead by E10 [14]. However, nearly one third of the Notch $1^{12 f / t m 1 C o n}$ embryos developed beyond E12.5 and died at various times during embryogenesis, including after birth (Table 2). Two Notch1 $1^{12 f / t m 1 \text { Con }}$ pups were found after birth, but none were observed after postnatal day 7 (Fig. 6, Table 2). Somite numbers in Notch1 ${ }^{12 f t t m 1 C o n}$ embryos varied from as low as Notch $1^{12 f l l b d}$ embryos to as high as wild type embryos (Table 1). Taken together, these results indicate that Notch $1{ }^{12 f}$ receptors present at a $50 \%$ dose in vivo, generate stronger Notch 1 signaling than Notch $1^{12 \mathrm{f}}$ in combination with Notch $1^{\text {lbd. This provides }}$ genetic evidence that Notch $1^{12 f}$ and Notch $11^{\text {lbd }}$ may functionally interact.

\section{Discussion and Conclusions}

In this paper we show that Notch1 signaling is greatly reduced in Notch1 ${ }^{12 f / l b d}$ ES cells and compound heterozygous embryos, but is significantly greater than in Notch $1^{\text {lbdllbd }}$ ES cells or homozygous embryos. The presence of the hypomorphic Notch 12 f allele allows vasculogenesis to proceed further and embryos to survive $\sim 1.5-2$ 


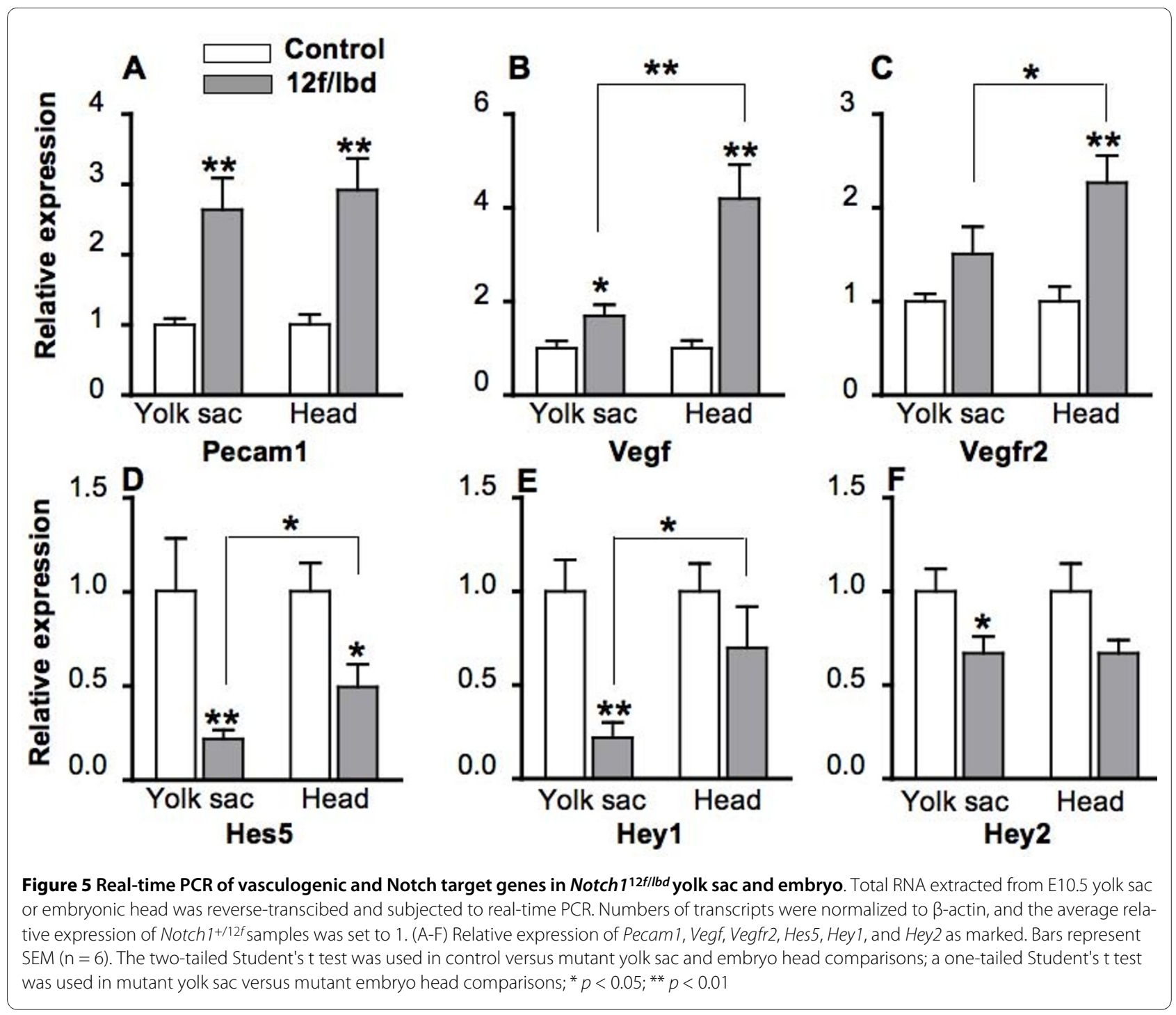

days longer. The vascular system develops early during mammalian embryogenesis. Initially, endothelial cell precursors differentiate and coalesce into a primitive network of undifferentiated blood vessels (the primary vascular plexus) in both the mammalian embryo and its extraembryonic membrane the yolk sac, in a process termed vasculogenesis [28]. Subsequently, the primary vascular plexus is remodeled into a highly organized and functionally competent vascular network in a process termed angiogenesis [29,30]. These processes are controlled by several signaling molecules, including vascular endothelial growth factor (VEGF) and its receptors [31], angiopoeitin 1 and its receptor Tie2 [32], Ephrin-B ligands and EphB receptors [33], TGF $\beta$ and its receptors [34], and Notch receptors and their ligands Delta and Jagged [25,26,35-38]. Defects in vasculogenesis are one of the major reasons that Notch1 null embryos die at midgestation $[13,26]$. Conditional mutation of Notch1 in vas- cular endothelial cells using the Tie2-Cre transgene showed that embryos lacking endothelial cell Notch1 die at $\sim$ E10.5 with profound vascular defects in placenta, yolk sac, and the embryo proper [27]. The Notch1 target genes Hey 1 and Hey 2 are also essential for embryonic vascular development [39]. A requirement for Notch signaling in the maintenance of vascular homestasis and the repression of endothelial cell proliferation is also indicated in adult mice by conditional deletion of RBP-JK in endothelial cells [40].

Interestingly, Notch112fllbd embryos allowed us to observe that vasculogenesis is regulated to different extents in yolk sac and embryo by Notch1 signaling. Thus, vascular defects in Notch112fllbd yolk sac were as severe as in Notch $1^{\text {lbd/lbd }}$ yolk sac, but vascular defects in Notch1 $1^{12 f l l b d}$ embryo heads were comparatively mild. The vasculogenic phenotype of Notch112f/lbd embryos was also milder than reported for Jagged1 or Notch1 or Notch1/4 

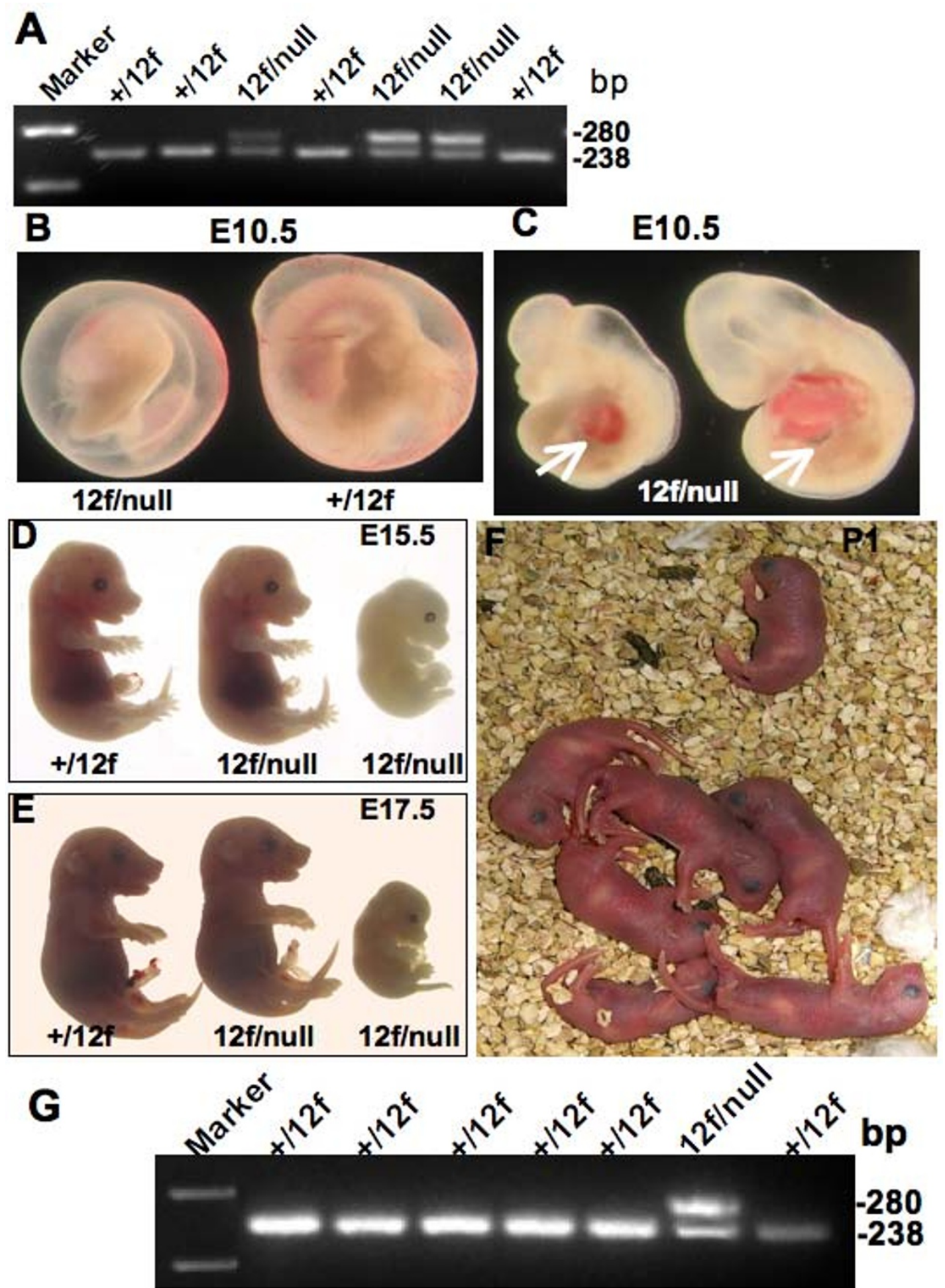

Figure 6 Notch 1 12f/tm1 1 on embryos survive longer than Notch112f/lbd embryos. (A) PCR genotype of an E9.5 litter showed the 280 bp PCR product

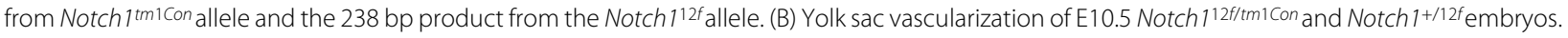
(C) Notch 1 12f/tmi Con embryos at E10.5 exhibit heamorrhaging around the heart (arrows). (D) Notch 1 12f//tm1 Con and control embryos at E15.5. One Notch $112 \mathrm{f} / \mathrm{tm} 1 \mathrm{Con}$ embryo was defective but the other had no obvious defects. (E) Notch 112f/tm1 Con and control embryos at E17.5. One Notch 1 12f/tmicon embryo was defective but the other had no obvious defects. (F) Photo of a litter on postnatal day 1 (P1) which included one pup identified as Notch $112 f /$ tm1 Con by PCR genotyping below. The pup was indistingishable but died within a few days. (G) PCR genotype of the P1 litter in panel F. 


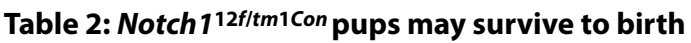

\begin{tabular}{|c|c|c|c|c|c|c|c|c|}
\hline \multirow[t]{2}{*}{ Stage } & \multicolumn{4}{|c|}{ 12f/12f $x+/$ tm1Con } & \multicolumn{4}{|c|}{$12 f / 12 f \times+/ l b d$} \\
\hline & Litters & Pups & $+/ 12 f$ & $\begin{array}{c}12 f / \\
\text { tm1Con }\end{array}$ & Litters & Pups & $+/ 12 f$ & $12 \mathrm{f} / \mathrm{lbd}$ \\
\hline E9.5 & 3 & 23 & 11 & 12 & 5 & 38 & 18 & 20 \\
\hline E10.5 & 3 & 23 & 13 & 10 & 8 & 70 & 37 & 33 \\
\hline E11.5 & 4 & 24 & 16 & 8 & 7 & 48 & 28 & 20 \\
\hline E12.5 & 5 & 25 & 21 & 4 & 8 & 33 & 28 & 5 \\
\hline E13.5 & 5 & 27 & 21 & 6 & 5 & 23 & 23 & 0 \\
\hline E15.5 & 3 & 13 & 9 & 4 & - & - & - & - \\
\hline E17.5 & 5 & 19 & 17 & 2 & - & - & - & - \\
\hline P1 & 6 & 25 & 23 & 2 & - & - & - & - \\
\hline Wean & 13 & 50 & 50 & 0 & 8 & 30 & 30 & 0 \\
\hline
\end{tabular}

Embryos (E9.5 -- E17.5), post-natal pups (P1) and mice after weaning were genotyped from crosses of Notch112f/12f female $\times$ Notch $1^{+/ t m 1 \text { Con }}$ male or crosses of Notch 1 12f/12f female $\times$ Notch $1^{+/ / b d}$ male. The data from Notch $1^{+/ 12 f} \times$ Notch $1^{12 f / l b d}$ crosses were previously published [12] but are included for ease of comparison.

null embryos [13,26,37], reflecting the presence of a low level of Notch1 signaling in Notch112fllbd compound heterozygotes. The reduced strength of Notch1 signaling was responsible for defective artery development in Delta-like 4 (Dll4) heterozygous embryos [38]. Hes5 and Hey1 are Notch1 target genes, and Notch1 downregulates expression of Hesr-1/Hey1 thereby enhancing expression of its target gene Vegfr2 in endothial cells [41]. In addition, Vegf is upregulated six-fold in Hey1/2 double knockout embryos [39]. Notch1 has also been proposed to regulate vasculogenesis and angiogenesis via induction of Ephrin-B2 [42,43] and Ang1 [44,45], and suppression of Vegfr-2/Kdr [41,46]. Consistent with this, we observed enhanced suppression of Vegfr2 and Vegf in Notch112fllbd yolk sac and embryo. However, we observed no change in the expression of Ang1, Tie2, Ephrin-B2 or Notch1 itself, although experiments in human endothelial cells indicate that Ang1 and Tie 2 are Notch1 target genes [44,45]. Ephrin-B2 was reported to respond to Notch4, but not to Notch1, through Delta-like 4 in differentiating HUVEC cells [43], so it was perhaps not surprising that Ephrin-B2 expression was unchanged in Notch112fllbd yolk sac or embryo. Thus, decreased Notch1 signaling may inhibit vascular development in yolk sac more than in embryos by inducing more Vegf and Vegfr 2 through generating less Hes5 and Hey 1 mRNA in yolk sac.

The prolonged embryonic development supported by the hypomorphic Notch $1{ }^{12 f}$ allele was only $~ 1.5-2$ days for Notch $1{ }^{12 f l l b d}$ embryos compared to Notch1 ${ }^{l b d l l b d}[8,12]$, or Notch1 null embryos [13,14]. By contrast Notch112f/12f,
Notch $1^{+/ l b d}$ or Notch1 $1^{+/}$heterozygotes are viable and fertile $[12-14,20]$. This suggests that Notch $1^{l b d}$ may interfere with Notch1 $12 f$ in a process termed negative complementation for Abruptex Notch mutants in Drosophila [47,48]. The basis of negative complementation is most commonly attributed to the products of the mutant alleles interacting physically [47]. Thus Notch $1^{\mathrm{lbd}}$ may either be dominant negative and inhibit Notch $1^{12 f}$ activity, or may not form a functional dimer or higher oligomer with Notch112f, if that is required for Notch1 to function. We prefer the latter hypothesis because there is no evidence to date that Notch1 ${ }^{\text {bd }}$ behaves as a dominant negative in Notch $1^{+/ l b d}$ heterozygotes $[8,12]$. Unfortunately, attempts to prove the existence of dimers or higher oligomers of Notch1 expressed at endogenous levels have so far been unsuccessful and previous attempts came to opposite conclusions. While two groups found that overexpressed Notch1 transfected into cultured cells may form dimers through the transmembrane domain or the extracellular domain EGF repeats, one group concluded that dimerization is necessary for Notch1 to signal [49], while the other concluded that Notch1 signals without the need for dimerization, and is present mainly as a monomer on the cell surface [50]. Both studies characterized transientlytransfected Notch1 expressed at much higher levels than endogenous Notch1, which might induce anomolous interactions.

If Notch $1^{\text {lbd }}$ reduces the effective amount of Notch1 ${ }^{12 f}$ to a level insufficient to sustain development, we reasoned that Notch $1{ }^{12 f}$ expressed in the context of a Notch1 
null background may function better. In fact, we found that a significant proportion of Notch $1^{12 f / t m 1 C o n}$ embryos survived beyond E12.5 and that some survived to birth. On the other hand, some compound heterozygous Notch 12fftm1Con embryos died at $\sim$ E11.5 with similar defects to Notch1 $12 f / l b d$. This indicates that Notch $1{ }^{12 f}$ at a dose of $50 \%$ functions at a threshold of Notch1 signaling strength that variably sustains embryogenesis through to birth - a stochastic effect or perhaps a genetic background effect, since Notch1+/12f and Notch1+/lbd mice were not extensively backcrossed to C57Bl/6. Nevertheless, the Notch1 signal strength generated by a single copy of Notch $1^{12 f}$ was intermediate between Notch $1^{12 f / 12 f}$ and Notch $12 f / l b d$, revealing the importance of maintaining a certain level of Notch1 signaling for mouse embryogenesis to proceed. Fig. 7 summarizes these findings in a diagram which describes a mini-allelic series of available Notch1 mutants. It includes the Notch1 processing point mutant Val1744Gly (Notch $1 v^{\prime} g / v^{\prime} g$ ) which has a phenotype very similar to, but slightly less penetrant than, a Notch1 null [15]. It also includes Notch $1^{+/ \text {null }}$ heterozygotes that have mild Notch1 signaling defects uncovered in competition assays [19] or by close examination of specific cell types $[18,20,21]$. Haploinsufficiency of NOTCH1 is the basis of aortic valve disease in humans [51]. We predict that Notch $1^{+/ l b d}$ and Notch1+/12f heterozygotes have slightly less Notch1 signaling than Notch1+ttm1Con and should display evidence of more extensive Notch1 signaling defects in particular cell types. The range of Notch1 mutant alleles available in the mouse should be helpful in identifying new in vivo functions for Notch1.

\section{Methods}

Mice

Mice carrying Notch1 lacking the $O$-fucose site in EGF12 (Notch1 ${ }^{12 f}$ ) and mice carrying Notch1 lacking the ligand binding domain $\left(\right.$ Notch $\left.1^{\text {lbd }}\right)$ were generated by gene tar-

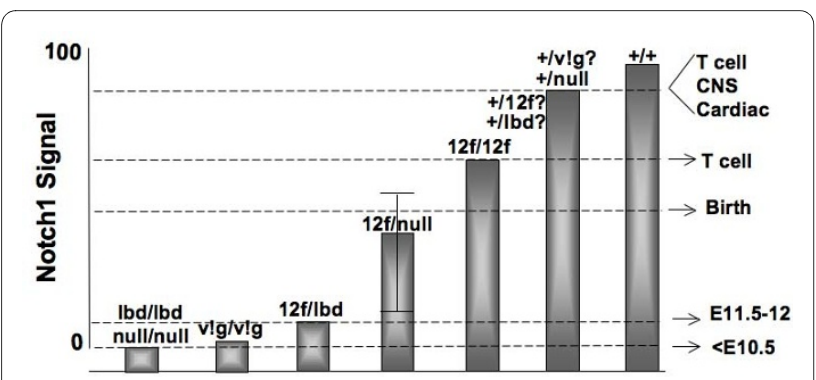

Figure 7 An allelic series of Notch1 mutants. Based on data reported herein and from the literature, the relative signaling strength of Notch 1 mutant alleles in various combinations with wild type or other Notch 1 mutant alleles is represented as discussed in the Discussion.

The consequences with respect to time of death of embryos with severe Notch1 signaling defects, or more subtle defects in T cell, CNS or cardiac development are noted. geting as previously described $[8,12]$. They were backcrossed 6-7 generations to $\mathrm{C} 57 / \mathrm{Bl} 6$ mice before being used in these experiments. Notch $1^{\text {l2fllbd }}$ embryos were obtained by crossing Notch $1^{12 f / 12 f}$ and Notch1+llbd mice. Embryos were collected from E9.5 and yolk sac DNA was genotyped by PCR using primers 5F: GTATGTATATGGGACTTGTAGGCAG and 6R: CTATGAGGGGTCACAGGACCAT, that give a $466 \mathrm{bp}$ product for the Notch $12 f$ allele and a 363 bp product for the wild type Notch1 allele; and primers 5F and 9R: CTTCATAACCTGTGGACGGGAG that give a 575 bp product for the Notch $1^{\text {lbd }}$ allele. The Notch1 null allele (Notch1tm1Con) encoding Notch1 lacking the transmembrane domain [14] backcrossed extensively to $\mathrm{C} 57 \mathrm{Bl} / 6$ was kindly provided by Cynthia Guidos, University of Toronto. Notch 1 12fttm1Con embryos were obtained by crossing Notch $1^{12 f / 12 f}$ and Notch1 $1^{+/ t m 1 C o n}$ mice and genotyped by PCR using primers neo-F: CTTGGGTGGAGAGGCTATTC and neo-R: AGGTGAGATGACAGGAGATC for the Notch $1^{t m 1 C o n}$ allele and primers loxF: GGCGAGCTCGAATTGATCC and 9R for Notch1 $12 f$ allele. Mice were housed under conventional barrier protection in accordance with Einstein and NIH guidelines. Protocols were approved by the Albert Einstein Animal Institute Committee.

\section{Embryonic stem cell isolation}

ES cells were isolated from E3.5 blastocysts as described [52], and genomic DNA was genotyped by PCR as described above. ES cells were routinely cultured on an SNL2 $\gamma$-irradiated feeder layer [53] in DMEM supplemented with $15 \%$ fetal bovine serum (Gemini, West Sacramento, CA), non-essential amino acids, L-glutamine, 1000 U ESGRO (Chemicon, Temecula, CA), 1\% $\beta$-mercaptoethanol, $25 \mathrm{mM}$ HEPES, penicillin $(50 \mathrm{U} / \mathrm{ml})$ and streptomycin $(50 \mu \mathrm{g} / \mathrm{ml})$. All reagents were from SpecialtyMedia, Lavellette, NJ. Before use in experiments, ES cells were passaged on gelatinized plates for 2-3 generations to remove feeder cells.

\section{Western blot analysis}

ES cells cultured on gelatinized plates were lysed in RIPA buffer (Upstate, Lake Placid, NY) containing complete protease inhibitor cocktail (Roche, Basel, Switzerland) for $30 \mathrm{~min}$ on ice and debris was removed by low speed centrifugation. Lysates were resolved by SDS-PAGE, transferred to polyvinyldifluoride (PVDF) membrane and probed with 8G10 anti-Notch1 mAb (Upstate, 57-557, 1:500, Lake Placid, NY) for full-length Notch1 or Val1744 Notch1 antibody (Cell Signaling Technology, Val1744, 1:1000, Beverly, MA) for cleaved, activated Notch1, followed by horseradish peroxidase(HRP)-conjugated secondary antibodies. Reactive bands were visualized with Enhanced Chemiluminescence Reagent (Amersham 
Pharmacia Biotech, Piscataway, NJ). $\beta$-tubulin-III specific antibody T8660 (Sigma Chemical Co., St. Louis, MO) was used as a loading control.

\section{Flow cytometry}

For cell surface Notch1 expression, 70-80\% confluent ES cells were dissociated from plates using phosphate-buffered saline (PBS)-based enzyme-free dissociation solution (SpecialtyMedia, Lavellette, NJ) for $10 \mathrm{~min}$ at $37^{\circ} \mathrm{C}$. After washing, ES cells $\left(5 \times 10^{5}\right)$ were incubated with 0.5 ug 8 G10 anti-Notch1 antibody in Hank's balanced salt solution containing 3\% bovine serum albumin Fraction $\mathrm{V}$ (Sigma Chemical Co., St. Louis, $\mathrm{MO}$ ), $1 \mathrm{mM} \mathrm{CaCl} 2$ and $0.05 \% \mathrm{Na}$ azide (HBSS/BSA) for $1 \mathrm{~h}$ at $4{ }^{\circ} \mathrm{C}$, washed and incubated in Alexa-488 conjugated anti-Hamster IgG $(1: 100)$ in HBSS/BSA in the dark (Invitrogen, Carlsbad, $\mathrm{CA}$ ) for $30 \mathrm{~min}$ at $4^{\circ} \mathrm{C}$. Immunofluorescence was analyzed on a FACSCalibur flow cytometer (BD Biosciences, San Diego, CA), gating on live cells determined by 7AAD staining. Data were analyzed using Flowjo software (Tree Star, San Carlos, CA).

\section{Notch co-culture signaling assay}

Notch signaling assays were performed in duplicate as previously described $[54,55]$. ES cells were plated at $2 \times$ $10^{5}$ cells per well of a six-well plate in ES medium, and cotransfected the next day with $0.2 \mu \mathrm{g}$ of TP1-luciferase Notch reporter plasmid and $0.05 \mu \mathrm{g}$ of Renilla luciferase reporter (pRL-TK; Promega, Madison, WI) along with 1.8 $\mu \mathrm{g}$ empty vector alone using FuGene 6 (Roche, Basel, Switzerland). At $16 \mathrm{~h}$ post-transfection, ES cells were overlaid with $1 \times 10^{6}$ rat Jagged1-expressing $\mathrm{L}$ cells (Jagged1/L), Delta1-expressing L cells (Delta1/L) or parental L cells [56]. At $48 \mathrm{~h}$ after transfection, firefly and Renilla luciferase activities were quantitated in cell lysates using a dual luciferase assay (Promega, Madison, WI). Ligand-dependent Notch activation was expressed as relative fold-activation of normalized luciferase activity stimulated by ligand/L cells compared to L cells.

\section{Notch ligand binding assay}

Soluble Notch ligand Delta1 with human Fc tag $[57,58]$ was prepared form HEK-293T cells expressing Delta1-Fc [17] cultured in $\alpha$-MEM containing 10\% FBS until 70 80\% confluence. The medium was changed to 293 SFM II serum-free medium (Invitrogen) and conditioned medium was collected after 3 days. Cellular debris was removed by low-speed centrifugation, the supernatant was filtered and stored at $4^{\circ} \mathrm{C}$. Soluble ligand concentration was determined by western blotting using HRP-conjugated anti-human IgG antibody (Jackson Immunoresearch, West Grove, PA). For the binding assay, ES cells on plates were dissociated using PBS-based Enzyme-free dissociation medium for $10 \mathrm{~min}$ at $37^{\circ} \mathrm{C}$, and the single cell suspension of ES was incubated with 2 $\mu \mathrm{g} / \mathrm{ml}$ Delta1-Fc in HBSS/BSA for $1 \mathrm{~h}$ at $4^{\circ} \mathrm{C}$, followed by incubation with 1:100 phycoerythrin (PE)-conjugated anti-human Fc antibody (Jackson Immunoresearch, West Grove, PA) for $30 \mathrm{~min}$ at $4^{\circ} \mathrm{C}$. After washing, live cells determined by gating on the 7-AAD negative population were analyzed on a FACS Calibur flow cytometer (BD Biosciences, San Jose, CA). Ligand binding ability was measured as mean fluorescence intensity (MFI) using Flowjo software (Tree Star, San Carlos, CA).

\section{Whole mount immunohistochemistry}

Embryos were collected on E9.5 and DNA from yolk sac was genotyped by PCR. Embryos were fixed in $4 \%$ paraformaldehyde (PFA) in PBS overnight at $4^{\circ} \mathrm{C}$, dehydrated through a methanol series, and bleached in $5 \% \mathrm{H}_{2} \mathrm{O}_{2} /$ methanol for $5 \mathrm{~h}$. Embryos were rehydrated and placed in PBSMT (PBS containing 3\% nonfat milk, 0.1\% Triton X100). After $2 \mathrm{~h}$, embryos were incubated with anti-mouse Pecam1 (1:200; BD Biosciences, San Jose, CA) in PBSMT overnight at $4{ }^{\circ} \mathrm{C}$. After 5 washes with PBSMT embryos were incubated in a 1:200 dilution of HRP-conjugated secondary antibody (Zymed, South San Francisco, CA) overnight. Embryos were washed 5 times in PBSMT and rinsed in PBT (PBS containing containing 0.2\% BSA, 0.1\% Triton X-100), followed developing with DAB kit (Vector Laboratories, Burlingame, CA). Finally, embryos were washed in PBT and postfixed in 4\% PFA, dehydrated through a methanol series and cleared in BABB (benzyl alcohol: benzyl benzoate - 1:2) in a glass Petri dish. Photos were taken in $\mathrm{PBS}$ or $\mathrm{BABB}$ using an inverted phase contrast microscope (Olympus IMT-2, Olympus America Inc., Center Valley, PA) and a Canon S40 camera with Tmount adaptor.

\section{Real-Time PCR}

Total RNA was extracted from yolk sac or embryo head using TRIZOL' reagent (Invitrogen, Carlsbad, CA) according to the manufacturer's instructions. Aliquots of $1 \mu \mathrm{g}$ RNA were digested by DNase I and cDNA was prepared using RNA PCR Kit ver. 3.0 (Takara Mirus Bio, Madison, WI) with oligo dT priming. Real-time PCR reactions with SybrGreen quantification were established with $1 / 20$ of each cDNA preparation in an Opticon2 DNA Engine (MJ Research, Cambridge, MA). Relative expression levels after normalization using $\beta$-actin were calculated using the $2^{-\Delta \Delta \mathrm{CT}}$ method ()([59] and confirmed by the absolute quantification method using standard curves. Primer pairs for real-time PCR were Ang1 (CATTCTTCGCTGCCATTCTG, GCACATTGCCCATGTTGAATC)[60], Pecam1 (GAGCCCAATC ACGTTTCA GTTT, TCCTTCCTGCTTCTTGCTA GCT) [60], Vegf (GGAGATCCTTCGAGGAGCACTT, GCGATTTAGCAGCAGAtATAagAa)[60], Tie2 (ATGTGGAaGTC- 
GAGAGGCGAT,

CGAATAGCCATCCACTATTG TCC)[60], Hey1 (TGAGCTGAGA AGGCTGGTAC, ACCCCAAACTCCGATAGTCC)[39], Hey2 (TGAGAAGACTAGTGCCAACAGC, TGGGC ATCA AAGTAGCCTTTA)[39], Ephrin-B2 (GCGGGATCCAGGA GATCCCCACTTGGACT, GTGCGCAACCTTCTCCTAAG)[39], Hes1 (AAGGCG GA CATTCTGGAAAT, GTCACCTCGTTCATGCACTC) [61]. Hes5 (TACCTGAAACACAGCAAAGC, GCTGGAGTGGTAAG CAG) [62] and $\beta$-actin (GTGGGCCGCTCT AGGCACCA, TGGCCTT AGGGTT CAGGGGG). All real-time PCR experiments were performed in duplicate from $\geq 4$ independent samples.

\section{Statistical analysis}

Statistical significance was calculated using the unpaired Student's $t$-test (two-tailed) using Graphpad Prism (GraphPad Software, Inc., San Diego, CA) unless otherwise noted.

\section{Authors' contributions}

PS conceived the project, obtained funding, participated in the design of experiments and analysis of data, and co-wrote the manuscript; CG partipated in the design of the experiments, performed or participated in all experiments, analysed data and co-wrote the paper. All authors read and approved the final version of the manuscript.

\section{Acknowledgements}

We thank Wen Dong for excellent technical assistance, Linchao Lu for helpful suggestions and Bin Zhou for helpful comments on the manuscript. This work was supported by NIH grant NCI RO1 95022 to PS and in part by NCl grant PO1 13330 to the Albert Einstein Cancer Center.

\section{Author Details}

'Department of Cell Biology, Albert Einstein College of Medicine, New York, NY-10461, USA and 2Beijing Institute of Radiation Medicine, Beijing, 100850, PR China

Received: 21 August 2009 Accepted: 29 March 2010

Published: 29 March 2010

\section{References}

1. Lai EC: Notch signaling: control of cell communication and cell fate. Development 2004, 131:965-73.

2. Schweisguth F: Regulation of notch signaling activity. Curr Biol 2004, 14:R129-38

3. Kopan R, llagan MX: The canonical Notch signaling pathway: unfolding the activation mechanism. Cell 2009, 137:216-33.

4. Moloney DJ, Shair LH, Lu FM, Xia J, Locke R, Matta KL, Haltiwanger RS: Mammalian Notch1 is modified with two unusual forms of O-linked glycosylation found on epidermal growth factor-like modules. $\mathrm{JBiO}$ Chem 2000, 275:9604-11.

5. Rebay I, Fleming RJ, Fehon RG, Cherbas L, Cherbas P, Artavanis-Tsakonas S: Specific EGF repeats of Notch mediate interactions with Delta and Serrate: implications for Notch as a multifunctional receptor. Cell 1991, 67:687-99.

6. Lieber T, Wesley CS, Alcamo E, Hassel B, Krane JF, Campos-Ortega JA, Young MW: Single amino acid substitutions in EGF-like elements of Notch and Delta modify Drosophila development and affect cell adhesion in vitro. Neuron 1992, 9:847-59.

7. Xu A, Lei L, Irvine KD: Regions of Drosophila Notch that contribute to ligand binding and the modulatory influence of Fringe. $\mathrm{J} \mathrm{Bio} / \mathrm{Chem}$ 2005, 280:30158-65
8. Ge C, Liu T, Hou X, Stanley P: In vivo consequences of deleting EGF repeats 8-12 including the ligand binding domain of mouse Notch1. BMC Dev Biol 2008, 8:48

9. Lei L, Xu A, Panin VM, Irvine KD: An O-fucose site in the ligand binding domain inhibits Notch activation. Development 2003, 130:6411-21.

10. Rampal R, Arboleda-Velasquez JF, Nita-Lazar A, Kosik KS, Haltiwanger RS: Highly conserved O-fucose sites have distinct effects on Notch1 function. J Biol Chem 2005, 280:32133-40.

11. Shi S, Ge C, Luo Y, Hou X, Haltiwanger RS, Stanley P: The threonine that carries fucose, but not fucose, is required for Cripto to facilitate Nodal signaling. J Biol Chem 2007, 282:20133-41.

12. Ge C, Stanley P: The O-fucose glycan in the ligand-binding domain of Notch1 regulates embryogenesis and T cell development. Proc Natl Acad Sci USA 2008, 105:1539-44.

13. Swiatek PJ, Lindsell CE, del Amo FF, Weinmaster G, Gridley T: Notch1 is essential for postimplantation development in mice. Genes Dev 1994, 8:707-19.

14. Conlon RA, Reaume AG, Rossant J: Notch1 is required for the coordinate segmentation of somites. Development 1995, 121:1533-45.

15. Huppert SS, Le A, Schroeter EH, Mumm JS, Saxena MT, Milner LA, Kopan R: Embryonic lethality in mice homozygous for a processing-deficient allele of Notch1. Nature 2000, 405:966-70.

16. Hadland BK, Huppert SS, Kanungo J, Xue Y, Jiang R, Gridley T, Conlon RA, Cheng AM, Kopan R, Longmore GD: A requirement for Notch1 distinguishes 2 phases of definitive hematopoiesis during development. Book A requirement for Notch 1 distinguishes 2 phases of definitive hematopoiesis during development City 2004, 104:3097-3105.

17. Stahl M, Uemura K, Ge C, Shi S, Tashima Y, Stanley P: Roles of Pofut1 and O-fucose in mammalian Notch signaling. J Biol Chem 2008, 283:13638-51.

18. Givogri MI, Costa RM, Schonmann V, Silva AJ, Campagnoni AT Bongarzone ER: Central nervous system myelination in mice with deficient expression of Notch1 receptor. J Neurosci Res 2002, 67:309-20.

19. Visan I, Tan JB, Yuan JS, Harper JA, Koch U, Guidos CJ: Regulation of T lymphopoiesis by Notch 1 and Lunatic fringe-mediated competition for intrathymic niches. Nat Immuno/ 2006, 7:634-43.

20. Loomes KM, Stevens SA, O'Brien ML, Gonzalez DM, Ryan MJ, Segalov M, Dormans NJ, Mimoto MS, Gibson JD, Sewell W, et al.: DIl3 and Notch1 genetic interactions model axial segmental and craniofacial malformations of human birth defects. Dev Dyn 2007, 236:2943-51.

21. Li Y, Takeshita K, Liu PY, Satoh M, Oyama N, Mukai Y, Chin MT, Krebs L, Kotlikoff MI, Radtke F, et al:: Smooth muscle Notch1 mediates neointimal formation after vascular injury. Circulation 2009, 119:2686-92.

22. Shi S, Stanley P: Protein O-fucosyltransferase 1 is an essential component of Notch signaling pathways. Proc Nat Acad Sci USA 2003, 100:5234-9

23. Oka C, Nakano T, Wakeham A, de la Pompa JL, Mori C, Sakai T, Okazaki S, Kawaichi M, Shiota K, Mak TW, et al.: Disruption of the mouse RBP-J kappa gene results in early embryonic death. Development 1995, 121:3291-301

24. Donoviel DB, Hadjantonakis AK, Ikeda M, Zheng H, Hyslop PS, Bernstein A: Mice lacking both presenilin genes exhibit early embryonic patterning defects. Genes Dev 1999, 13:2801-10.

25. Swift MR, Weinstein BM: Arterial-venous specification during development. Circ Res 2009, 104:576-88

26. Krebs LT: Notch signaling is essential for vascular morphogenesis in mice. Genes Dev 2000, 14:1343-1352.

27. Limbourg FP, Takeshita K, Radtke F, Bronson RT, Chin MT, Liao JK: Essential role of endothelial Notch1 in angiogenesis. Circulation 2005, 111:1826-32

28. Risau W, Flamme I: Vasculogenesis. Annu Rev Cell Dev Biol 1995, 11:73-91.

29. Flamme I, Frolich T, Risau W: Molecular mechanisms of vasculogenesis and embryonic angiogenesis. J Cell Physiol 1997, 173:206-10.

30. Risau W: Mechanisms of angiogenesis. Nature 1997, 386:671-4

31. Carmeliet P, Ferreira V, Breier G, Pollefeyt S, Kieckens L, Gertsenstein M, Fahrig M, Vandenhoeck A, Harpal K, Eberhardt C, et al.: Abnormal blood vessel development and lethality in embryos lacking a single VEGF allele. Nature 1996, 380:435-9.

32. Suri C, Jones PF, Patan S, Bartunkova S, Maisonpierre PC, Davis S, Sato TN Yancopoulos GD: Requisite role of angiopoietin-1, a ligand for the TIE2 receptor, during embryonic angiogenesis. Cell 1996, 87:1171-80. 
33. Wang HU, Chen ZF, Anderson DJ: Molecular distinction and angiogenic interaction between embryonic arteries and veins revealed by ephrinB2 and its receptor Eph-B4. Cell 1998, 93:741-53.

34. Oshima M, Oshima H, Taketo MM: TGF-beta receptor type II deficiency results in defects of yolk sac hematopoiesis and vasculogenesis. Dev Biol 1996, 179:297-302.

35. Shawber CJ, Kitajewski J: Notch function in the vasculature: Insights from zebrafish, mouse and man. Bioessays 2004, 26:225-234.

36. Krebs LT: Haploinsufficienct lethality and formation of arteriovenous malformations in Notch pathway mutants. Genes Dev 2004, 18:2469-2473.

37. Xue Y, Gao X, Lindsell CE, Norton CR, Chang B, Hicks C, Gendron-Maguire $M$, Rand EB, Weinmaster G, Gridley T: Embryonic lethality and vascular defects in mice lacking the Notch ligand Jagged1. Hum Mol Genet 1999, 8:723-30.

38. Duarte A: Dosage-sensitive requirement for mouse DII4 in artery development. Genes Dev 2004, 18:2474-2478.

39. Fischer A, Schumacher N, Maier M, Sendtner M, Gessler M: The Notch target genes Hey1 and Hey2 are required for embryonic vascular development. Genes Dev 2004, 18:901-11

40. Dou GR, Wang YC, Hu XB, Hou LH, Wang CM, Xu JF, Wang YS, Liang YM, Yao LB, Yang AG, et al:: RBP-J, the transcription factor downstream of Notch receptors, is essential for the maintenance of vascular homeostasis in adult mice. FASEB J 2008, 22:1606-17.

41. Taylor KL, Henderson AM, Hughes CC: Notch activation during endothelial cell network formation in vitro targets the basic $\mathrm{HLH}$ transcription factor HESR-1 and downregulates VEGFR-2/KDR expression. Microvasc Res 2002, 64:372-83.

42. Lawson ND: Notch signaling is required for arterial-venous differentiation during embryonic vascular development. Development 2001, 128:3675-3683.

43. Shawber CJ, Das I, Francisco E, Kitajewski J: Notch signaling in primary endothelial cells. Ann N Y Acad Sci 2003, 995:162-70.

44. Morrow D, Cullen JP, Cahill PA, Redmond EM: Cyclic strain regulates the Notch/CBF-1 signaling pathway in endothelial cells: role in angiogenic activity. Arterioscler Thromb Vasc Biol 2007, 27:1289-96.

45. Morrow D, Cullen JP, Cahill PA, Redmond EM: Ethanol stimulates endothelial cell angiogenic activity via a Notch- and angiopoietin-1dependent pathway. Cardiovasc Res 2008, 79:313-21.

46. Suchting S, Freitas C, le Noble F, Benedito R, Breant C, Duarte A, Eichmann A: The Notch ligand Delta-like 4 negatively regulates endothelial tip cell formation and vessel branching. Proc Natl Acad Sci USA 2007, 104:3225-30.

47. Foster GG: Negative complementation at the notch locus of Drosophila melanogaster. Genetics 1975, 81:99-120.

48. Portin P: Allelic negative complementation at the Abruptex locus of Drosophila melanogaster. Genetics 1975, 81:121-33.

49. Sakamoto K, Chao WS, Katsube K, Yamaguchi A: Distinct roles of EGF repeats for the Notch signaling system. Exp Cell Res 2005, 302:281-91.

50. Vooijs M, Schroeter EH, Pan Y, Blandford M, Kopan R: Ectodomain shedding and intramembrane cleavage of mammalian Notch proteins is not regulated through oligomerization. J Biol Chem 2004, 279:50864-73.

51. Garg V: Molecular genetics of aortic valve disease. Curr Opin Cardiol 2006, 21:180-4.

52. Roach ML, McNeish JD: Methods for the isolation and maintenance of murine embryonic stem cells. Methods Mol Biol 2002, 185:1-16.

53. loffe E, Stanley P: Mice lacking N-acetylglucosaminyltransferase activity die at mid-gestation, revealing an essential role for complex or hybrid N-linked carbohydrates. Proc Natl Acad Sci USA 1994, 91:728-32.

54. Chen J, Moloney DJ, Stanley P: Fringe modulation of Jagged1-induced Notch signaling requires the action of beta 4galactosyltransferase-1. Proc Natl Acad Sci USA 2001, 98:13716-21.

55. Shi S, Stahl M, Lu L, Stanley P: Canonical Notch signaling is dispensable for early cell fate specifications in mammals. Mol Cell Biol 2005, 25:9503-8.

56. Hicks C, Johnston SH, diSibio G, Collazo A, Vogt TF, Weinmaster G: Fringe differentially modulates Jagged 1 and Delta 1 signalling through Notch1 and Notch2. Nat Cell Biol 2000, 2:515-20.

57. Wang S, Sdrulla AD, diSibio G, Bush G, Nofziger D, Hicks C, Weinmaster G, Barres BA: Notch receptor activation inhibits oligodendrocyte differentiation. Neuron 1998, 21:63-75.
58. Hicks C, Ladi E, Lindsell C, Hsieh JJ, Hayward SD, Collazo A, Weinmaster G: A secreted Delta1-Fc fusion protein functions both as an activator and inhibitor of Notch1 signaling. J Neurosci Res 2002, 68:655-67.

59. Livak KJ, Schmittgen TD: Analysis of relative gene expression data using real-time quantitative PCR and the 2(-Delta Delta C(T)) Method. Methods 2001, 25:402-8

60. Shih SC, Robinson GS, Perruzzi CA, Calvo A, Desai K, Green JE, Ali IU, Smith $L E$, Senger DR: Molecular profiling of angiogenesis markers. Am J Pathol 2002, 161:35-41.

61. Yin D, Xie D, Sakajiri S, Miller CW, Zhu H, Popoviciu ML, Said JW, Black KL, Koeffler HP: DLK1: increased expression in gliomas and associated with oncogenic activities. Oncogene 2006, 25:1852-61.

62. Ikawa T, Kawamoto H, Goldrath AW, Murre C: E proteins and Notch signaling cooperate to promote $\mathrm{T}$ cell lineage specification and commitment. J Exp Med 2006, 203:1329-42.

doi: $10.1186 / 1471-213 X-10-36$

Cite this article as: Ge and Stanley, Effects of varying Notch1 signal strength on embryogenesis and vasculogenesis in compound mutant heterozygotes BMC Developmental Biology 2010, 10:36

\section{Submit your next manuscript to BioMed Central and take full advantage of:}

- Convenient online submission

- Thorough peer review

- No space constraints or color figure charges

- Immediate publication on acceptance

- Inclusion in PubMed, CAS, Scopus and Google Scholar

- Research which is freely available for redistribution

Submit your manuscript at www.biomedcentral.com/submit
C Biomed Central 